\title{
Mesangioproliferative glomerulonephritis associated with retinitis pigmentosa
}

\author{
P A KALRA AND J H TURNEY \\ From the Renal Unit, General Infirmary at Leeds, Great George Street, Leeds LS1 3EX
}

SUMmaRY Two patients with retinitis pigmentosa and glomerulonephritis are described. The patients were unrelated and had identical renal lesions, mesangioproliferative glomerulonephritis. This may not be a chance association, and simple dip-stick urine analysis in patients with primary retinal degenerations may lead to earlier identification of renal disease.

The association between primary retinal degeneration and renal disease has been well described, ${ }^{1-6}$ but usually in the context of inherited syndromes with multiple abnormalities, such as the Laurence-MoonBiedl syndrome, which are often apparent at or soon after birth.

We describe two unrelated patients who were diagnosed as having retinitis pigmentosa during their third decade and who had different renal presentations of the same underlying glomerular lesion in adult life. Neither had a family history of blindness or nephritis, and though the patient in case 2 also had a disorder of cardiac conduction and neuronal deafness there were no features suggestive of a recognised syndrome in either case.

\section{Case reports}

CASE 1

A 65-year-old woman had been registered as partially sighted for many years, her ophthalmological diagnosis being retinitis pigmentosa. She presented in 1979 with proteinuria, hypertension, and microscopic haematuria. On demonstration of highly selective proteinuria a presumptive diagnosis of minimal change glomerulonephritis was made. The proteinuria and oedema remitted with diuretics, cyclophosphamide, and steroids, which were withdrawn after nine months.

She was referred to the renal unit five years later with a relapse of her nephritis. The 24-hour urinary protein excretion was $7 \mathrm{~g}$ and creatinine clearance was $18 \mathrm{ml} / \mathrm{min}$. Urine microscopy demonstrated an active sediment, with casts and red cells; a test for Correspondence to Dr P A Kalra, 80 Islington Street, Ponsonby, Auckland, New Zealand. antinuclear factor (ANF) was negative, and immunoglobulins were within the normal ranges. A diuresis was obtained with a salt-poor albumin infusion and diuretics, and she was also restarted on prednisolone. Renal biopsy was performed, and histological appearances were those of mesangial proliferative glomerulonephritis, a diagnosis supported by electron microscopy.

A progressive decline in the level of proteinuria over the next six months was accompanied by a considerable improvement in renal function. She is at present well, requiring no immunosuppressive therapy.

CASE 2

A 26-year-old man had been diagnosed as hypertensive at the time of total hip replacement for secondary osteoarthrosis. Blood pressure required treatment with nadolol, minoxidil, and nifedipine for control.

Five years later he was admitted to the coronary care unit with severe central chest pain unassociated with arrhythmia. He was a stocky, unusual looking man with hypertrichosis and a broad, flat facies; he appeared to be of normal intelligence. Chromosome analysis was normal. Serial ECG recordings, though demonstrating type A Wolfe-Parkinson-White syndrome and left ventricular hypertrophy, provided no evidence of myocardial infarction, and there was no increase of serum enzymes. After a second episode of pain, normal coronary arteries were shown at angiography. He was noted to have chronic renal failure with a creatinine clearance of $67 \mathrm{ml} / \mathrm{min}$. The 24-hour urinary protein excretion was $5.4 \mathrm{~g}$, antinuclear factor (ANF) negative, serum IgA and IgG both low, and the kidneys were shrunken with reduced cortical thickness on ultrasound scan. Renal biopsy demon- 
strated mesangioproliferative glomerulonephritis, and immunofluorescence confirmed mesangial $\operatorname{IgA}$ deposition. During the same admission to hospital the patient's complaints of deteriorating hearing and vision were investigated, and both sensorineural deafness and retinitis pigmentosa were diagnosed.

The patient at present has well controlled hypertension, but renal function continues to deteriorate.

\section{Discussion}

There have been several descriptions of hereditary nephritis coexisting in families with retinitis pigmentosa. Meier and Hess' described the first such family, in which the renal disease was juvenile nephronophthisis, and Sarles et al. ${ }^{2}$ reported a family in which the renal pathology was consistent with chronic interstitial nephritis. Other rare hereditary syndromes describe renal disease and retinitis pigmentosa occurring in association with multiple abnormalities, such as tubular atrophy and glomerular sclerosis in mitochondrial cytopathy ${ }^{3}$ and in Schwachman's syndrome, where renal abnormalities include aminoaciduria and nephrocalcinosis. ${ }^{+}$ However, the only description of glomerulonephritis coexisting with primary retinal degeneration is in the Laurence-Moon-Biedl syndrome." Though in this rare, recessively inherited condition obesity, pigmentary retinopathy, hypogonadism, polydactyly, and mental retardation are often the more prominent features, uraemia is thought to be the commonest cause of death. The most frequent renal lesion appears to be a form of medullary cystic disease, ${ }^{6}$ but mesangial glomerulonephritis, glomerulosclerosis, and obstructive uropathy have all been reported.

Our two cases also had mesangioproliferative glomerulonephritis coexisting with retinitis pigmentosa. However, the patients were unrelated, had no relevant family history of ocular or renal disease and no features to suggest a congenital syndrome of multiple abnormalities. Their renal disease also presented much later in life, in the sixth and fourth decades respectively. We consider that these cases represent more than just chance association, and that routine urine analysis in patients with retinitis pigmentosa may identify more cases with coexisting glomerulonephritis, which can then receive appropriate treatment at a much earlier stage.

If a relationship does indeed exist between retinitis pigmentosa and mesangioproliferative glomerulonephritis, then it is possible that the conditions share the same underlying pathogenetic process. Kandori et $a l .^{\prime}$ have described unilateral pigmentary degeneration occurring after retinal vascular hypoperfusion.
Although it would be quite plausible for proliferative glomerular lesions to result from ischaemia, in the majority of cases of retinitis pigmentosa the choroidal circulation appears to be normal in the earlier stages, ${ }^{x}$ even though the choriocapillaris is seen to become irregular and unfilled as the condition progresses. ${ }^{\prime}$

Perhaps a specific enzyme of critical importance to the function of both retina and kidney is deficient. This has been suggested in certain syndromes, thought to have a genetic basis, in which retinal degeneration and renal disease are prominent features, such as familial renal-retinal dystrophy"1" (the renal lesion in this syndrome is a form of medullary cystic disease resembling nephronophthisis). Alternatively, disordered metabolism of a fatty acid important to both retina and glomerulus may be involved. This has been postulated in the aetiology of the retinal degeneration seen in Refsum's disease, where defective vitamin A production may be relevant." We acknowledge that chromosome analysis of our two patients may well prove to be of interest.

We acknowledge the help of Mrs Carolyn Czenkusz, who typed the manuscript.

\section{References}

1 Meier DA, Hess JW. Familial nephropathy with retinitis pigmentosa. Am J Med 1965; 39: 58-69.

2 Sarles HE, Rodin AE, Poduska PR, Smith GH, Fish JC, Remmers AR Jr. Hereditary nephritis, retinitis pigmentosa and chromosomal abnormalities. Am J Med 1968; 45: 312-21.

3 Egger J, Lake BD, Wilson J. Mitochondrial cytopathy-a multisystem disorder with ragged red fibres on muscle biopsy. Arch Dis Child 1981; 56: 741-52.

4 Aggett PJ, Cavanagh NP, Matthew DJ, Pincott JR, Sutcliffe J, Harries JT. Shwachman's syndrome. Arch Dis Child 1980; 55: 331-47.

5 Hurley RM, Dery P, Nogrady MB, Drummond KN. The renal lesion of the Laurence-Moon-Biedl syndrome. J Pediatr 1975; 87: 206-9.

6 Bluett NH, Chantler C, Singer JD, Saxton HM. Congenital renal abnormalities in the Laurence-Moon-Biedl syndrome. Arch Dis Child 1977; 52: 968-70.

7 Kandori F, Tamai A, Watanabe T, Kurimoto S. Unilateral pigmentary degeneration of the retina. Am J Ophthalmol 1968; 66: 1091-101.

8 Cogan DG. Pathology. In: Symposium-Primary chorioretinal aberrations with night blindness. Ophthalmology 1950; 54: 629-61.

9 Weinstein GW, Maumanee AE, Hyvarinen L. On the pathogenesis of retinitis pigmentosa. Ophthalmologica 1971: 162: $82-97$.

10 Senior B. Familial renal-retinal dystrophy. Am J Dis Child 1973; 125: 442-7.

11 Toussaint D, Danis P. An ocular pathologic study of Refsum's syndrome. Am J Ophthalmol 1971; 72: 342-7.

Accepted for publication 20 January 1987. 